\title{
A Gamma and Neutron Counter of Undoped CsI Crystals with WLSFiber Readout for the KOTO Experiment
}

\author{
Naoki Kawasaki for the КОТО collaboration * \\ Department of Physics, Kyoto University, Kyoto 606-8502, Japan \\ E-mail: kawasakidscphys.kyoto-u.ac.ip
}

The KOTO experiment at J-PARC aims to observe the $K_{L}^{0} \rightarrow \pi^{0} v \bar{v}$ decay, which is a rare direct $\mathrm{CP}$-violating process and a powerful probe to search for new physics beyond the Standard Model. In this experiment, there are remaining neutrons around the narrow $K_{L}$ beam, called "halo neutrons". $\pi^{0}$ 's generated by the interaction of halo neutrons with the KOTO detector become a serious background. To suppress and estimate this neutron background, we developed a gamma and neutron counter, called Neutron Collar Counter (NCC). NCC modules are made of undoped CsI crystals, divided into 3 parts along beam direction and read with wavelength shifting fibers. NCC was designed to veto halo neutron backgrounds by detecting the energy deposited in neutron interactions. NCC was also designed to distinguish neutron events from other events from $K_{L}$ decays using the difference of shower shapes, and to measure the flux and energy of halo neutrons. Construction of NCC was finished in Nov. 2012. It was installed inside an almost complete KOTO detector in December 2012. This paper describes the concepts behind NCC, its design and the results of its performance characterization.

2013 Kaon Physics International Conference,

29 April-1 May 2013

University of Michigan, Ann Arbor, Michigan - USA

\footnotetext{
*Speaker.
} 


\section{Introduction}

\subsection{KOTO experiment}

The KOTO experiment aims to achieve the first observation of the decay $K_{L} \rightarrow \pi^{0} v \bar{v}$ using an upgraded KEK-E391a detector and a high intensity $K_{L}^{0}$ beam line constructed at the Japan Proton Accelerator Research Complex (J-PARC). The $K_{L}^{0} \rightarrow \pi^{0} v \bar{v}$ decay is a rare direct CP-violating process and its branching ratio is proportional to $\eta^{2}$, where eta represents the complex phase of CKM matrix. If there is any contribution from a new particle in the loop diagram of this decay mode, the branching ratio may vary from the Standard Model prediction of $(2.4 \pm 0.4) \times 10^{-11}$ [四]. As such, this decay mode is a good probe for a new physics.

Fig. $\square$ shows a side view of the KOTO detector. To identify the $K_{L}^{0} \rightarrow \pi^{0} v \bar{v}$ decay, we take the following strategy. First, the two $\gamma$ 's from the $\pi^{0}$ in the $K_{L}$ decay are detected by a CsI calorimeter and their energies and positions are measured. Constraining the two photons to come from a $\pi^{0}$, we can reconstruct the decay vertex and momentum of the $\pi^{0}$. Various kinematic cuts are applied to suppress backgrounds. Second, hermetic veto counters surround the decay volume to ensure there is nothing except $2 \gamma$ 's from a $\pi^{0}$. The calorimeter and the veto counters surrounding the decay volume are placed inside a pressure vessel evacuated down to $10^{-5} \mathrm{~Pa}$ to suppress backgrounds.
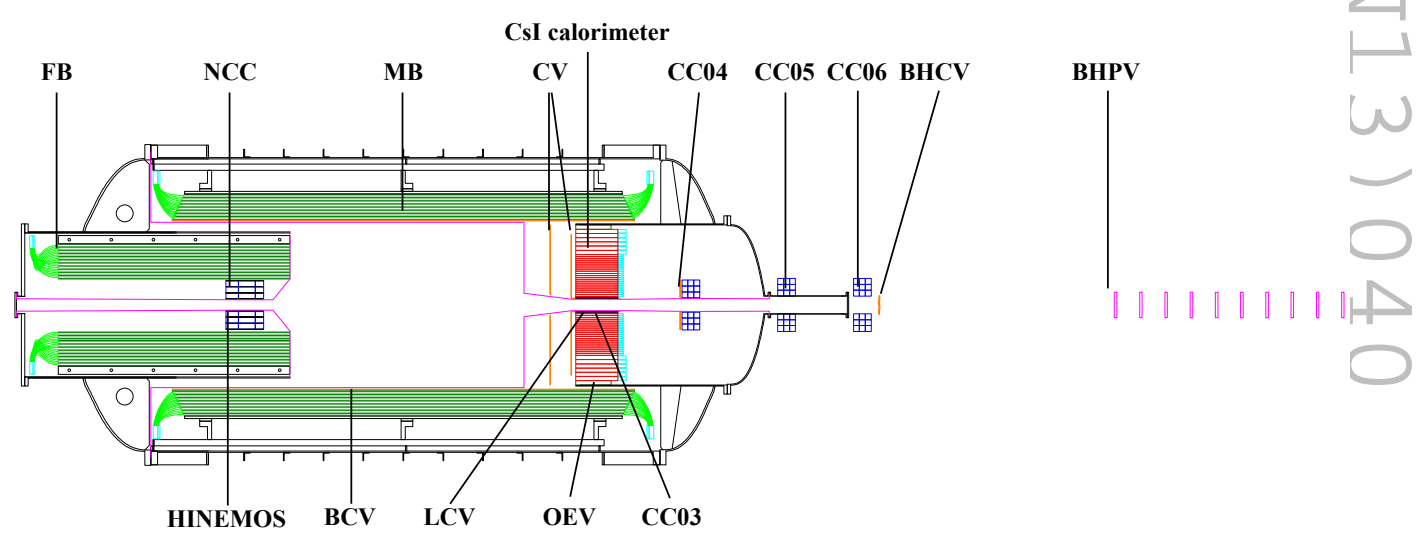

Figure 1: A side view of the KOTO detector. Hermetic veto counters surrounding the decay volume are shown. Neutron Collar Counter(NCC), discussed in this paper, is located at the entrance of the decay volume.

\subsection{Halo neutron background}

In the E391a experiment, one of the major backgrounds came from $\pi^{0}$ 's generated by halo neutrons hitting the $\mathrm{CC} 02$ veto counter, which surrounded the beam hole at the entrance of decay volume as shown in Fig. $\square$ (the $\mathrm{CC} 02$ counters was located in a position similar to that of the NCC.).

Most of these events can be rejected by their vertex positions as shown in Fig. B, which is the distribution of the reconstructed $\pi^{0}$ transverse momentum versus its vertex z position for the final event selection of E391a experiment. The cluster of events on the left of the signal box, at lower 
$\mathrm{z}$ positions, is due to halo neutron background. The vertex can be reconstructed toward larger $\mathrm{z}$ positions if the $\gamma$ energy is mis-measured, either from shower leakage or photo-nuclear interactions in the calorimeter. This was estimated to be one of the main background sources in E391a though no event was observed in the signal box.

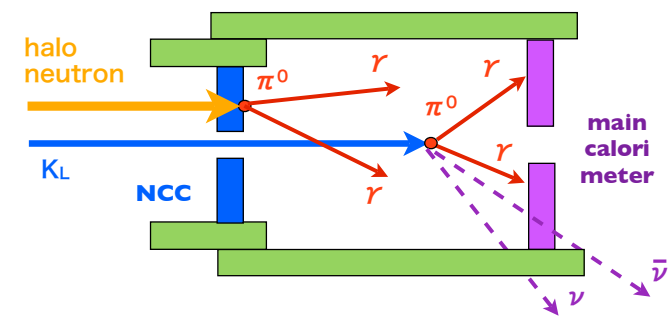

Figure 2: A schematic view of $K_{L} \rightarrow \pi^{0} v \bar{v}$ events and halo neutron backgrounds.

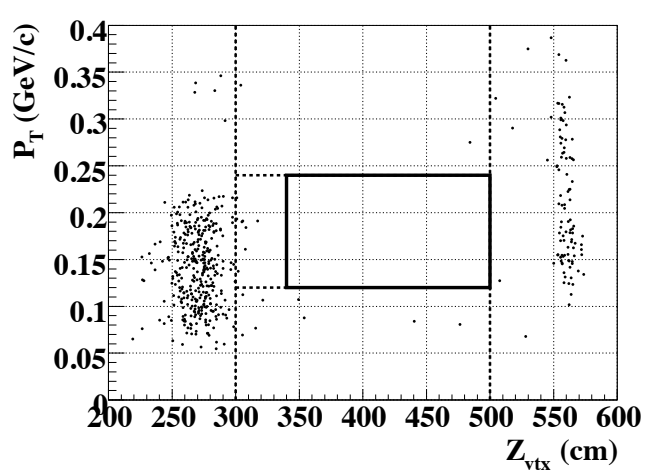

Figure 3: Final result of the E391a experiment. The vertical axis shows the reconstructed transverse momentum of the $\pi^{0}$. The horizontal axis shows the reconstructed $\mathrm{Z}$ position of the $\pi^{0}$. The region bounded by the solid line shows the signal box. The cluster on the left is the events produced by neutrons hitting $\mathrm{CCO} 2$ [2].

\section{Neutron Collar Counter(NCC)}

\subsection{Detector Design}

$\mathrm{NCC}$ is located at the entrance of the decay volume and surrounds the narrow $K_{L}$ neutral beam, as an upgrade of E391a CC02 veto counter. A front and a side view of NCC are shown in Fig. 6 .

We designed NCC to play two important roles against halo neutron background, one is background reduction and the other is background estimation. NCC consists of 48 inner modules and 8 additional outer modules. All these modules consist of undoped CsI crystals. CsI crystals have a relatively long interaction length $\left(\lambda_{I}=39 \mathrm{~cm}\right)$ and short radiation length $\left(X_{0}=1.9 \mathrm{~cm}\right)$. Thus CsI is advantageous to suppress $\pi^{0}$ generation by neutron interactions and to stop $2 \gamma$-rays from generated $\pi^{0}$ decay.

From the point of view of halo neutron measurement, the $K_{L}$ decay products (especially $\gamma$ rays) become backgrounds. At the NCC position, single counting rate by $K_{L}$ decay is $\sim 100$ times larger than that of halo neutrons. To distinguish neutrons from other particles from $K_{L}$ decay, each module consists of 3 optically separated CsI crystals ( Front, Middle, and Rear). The front and side views of the NCC modules are shown in Fig. 6. According to a detector simulation, we can suppress the contamination of $K_{L}$ decays from halo neutron events by more than $50 \%$.

It is not possible to attach PMTs directly onto the inner crystals, thus we adopted a readout of scintillation light via wavelength shifting fibers. We use Kuraray PMP Fibers, whose absorption 
and emission spectrum matches the CsI emission spectrum and the quantum efficiency of PMTs (Fig. [5).

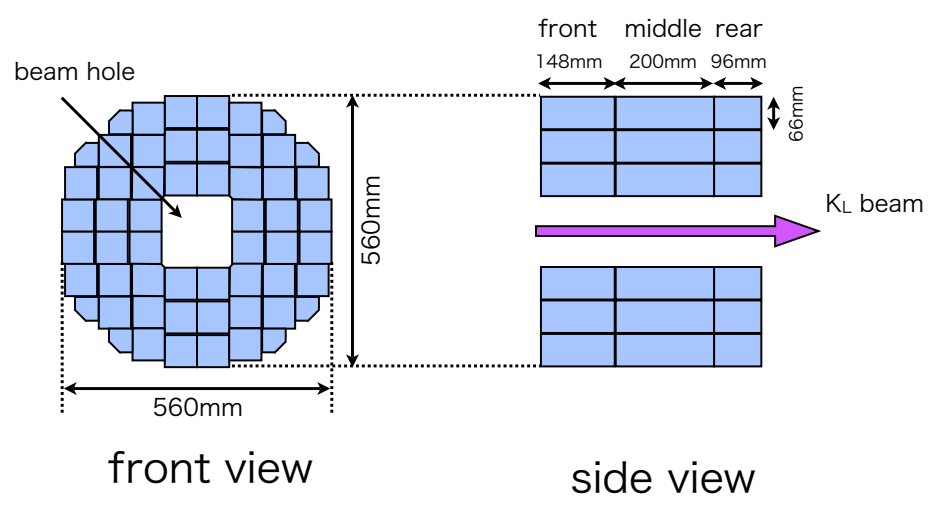

Figure 4: Front view (left) and side view (right) of the NCC

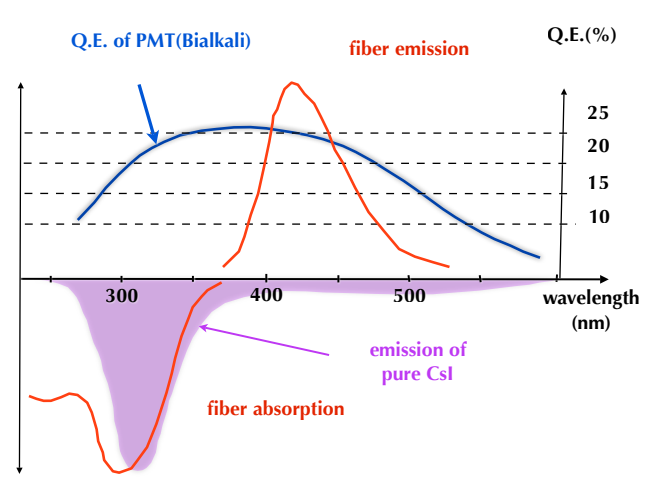

Figure 5: Emission spectrum of pure CsI, absorption and emission spectrum of PMP fiber, and quantum efficiency of PMTs.

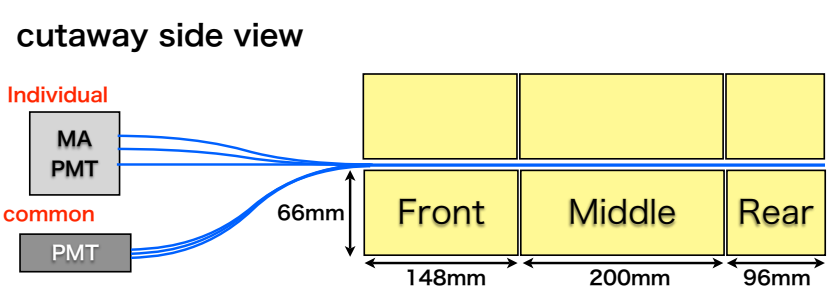

top view

Individual Common

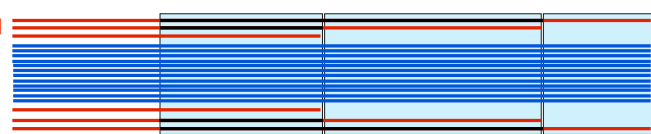

Figure 6: Cutaway and top view of NCC modules. The upper figure is cutaway side view, and the lower figure is top view.

\subsection{Detector Performance}

We produced the NCC modules from Apr. 2012 to Aug. 2012. In order to veto the background effectively, we need the NCC detector to be sensitive to energy deposition at the $1 \mathrm{MeV}$ level and to achieve a light yield of 4.5 p.e./MeV with a uniformity within $20 \%$. We checked the performance of all modules using cosmic rays. The setup is shown in Fig. $\square$. We measured the light yield of front, middle, and rear sections individually ( Fig. [إ), and calculated the uniformity of each module (Fig. Q Q). These results show that all modules pass the requirements set above.

We finished the stacking and installation of NCC inside the KOTO detector in December 2012. During the December 2012 engineering run, the NCC could identify cosmic ray events using $\mu$ - 


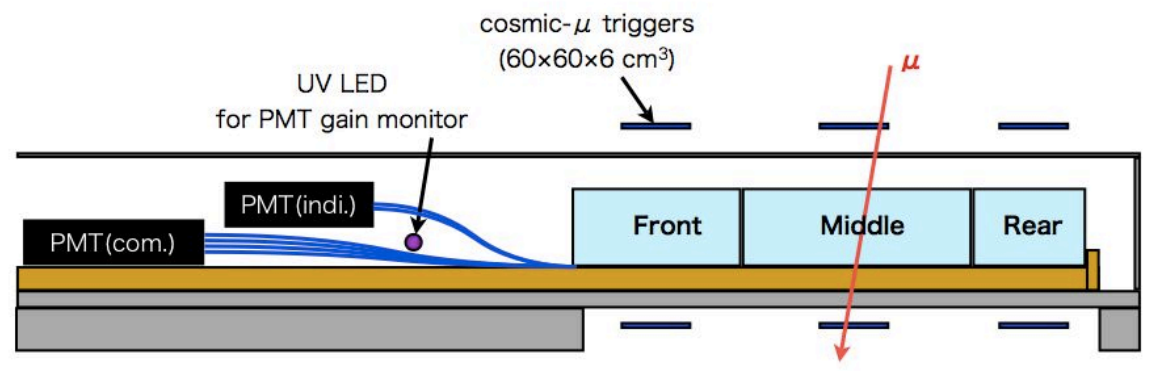

Figure 7: An overview of the setup of light yield measurement by cosmic rays. There are three pairs of cosmic- $\mu$ triggers made of plastic scintillators to measure the light yield of each crystal individually.

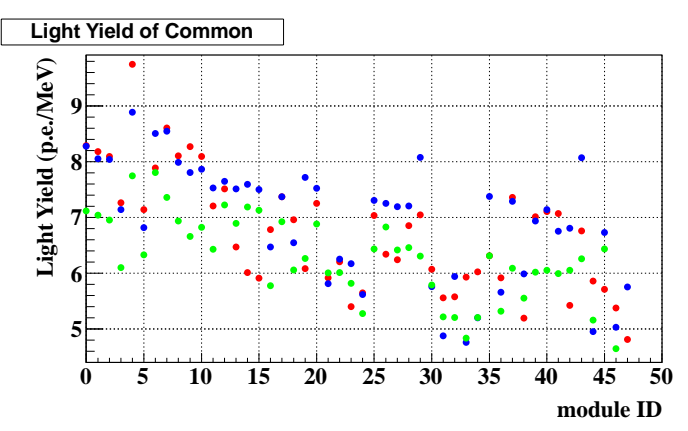

Figure 8: Light yield of all modules. red : light yield of front part, green : light yield of middle part, blue : Figure 9: Uniformity of light yield of all modules . light yield of rear part

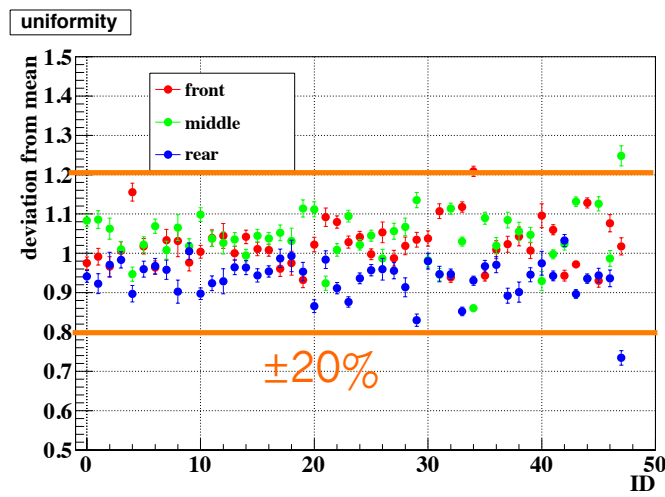

Light yield of each part is normalized by the average of light yield of front, middle, and rear part.

tracking, as shown in Fig. 10 , and obtain ADC energy spectra for all channels. By fitting such ADC spectra with a Landau function (Fig.WI), we can calibrate the energy scale of all channels with less than $1 \%$ error.

\section{Summary}

We developed a new gamma and neutron counter made of undoped CsI crystals for the KOTO detector, called Neutron Collar Counter. This detector is placed at the entrance of the KL decay volume and is designed to both reduce the $\pi^{0} \mathrm{~s}$ background from interaction of halo neutrons with the detector material and to measure the flux and spectrum of halo neutrons.

NCC production was finished in Nov.2012 and the required light yield and uniformity were achieved for all the modules. NCC was installed inside the KOTO detector for the Dec. 2012 engineering run. A calibration method using cosmic-ray tracking was established and commissioned for future physics runs. 


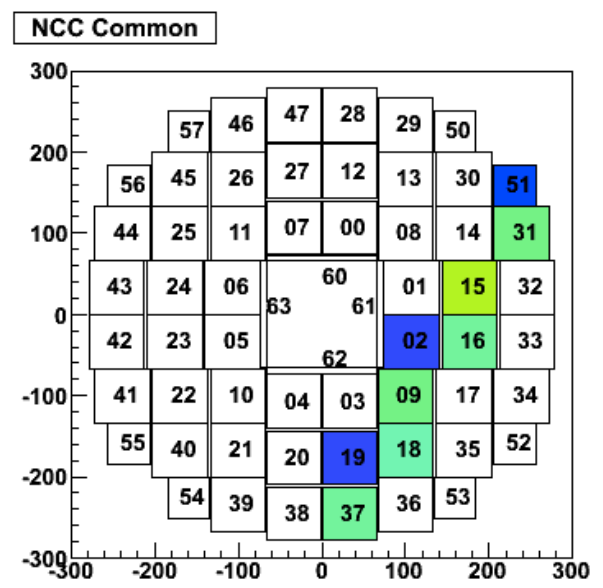

Figure 10: A event display of a cosmic- $\mu$ track

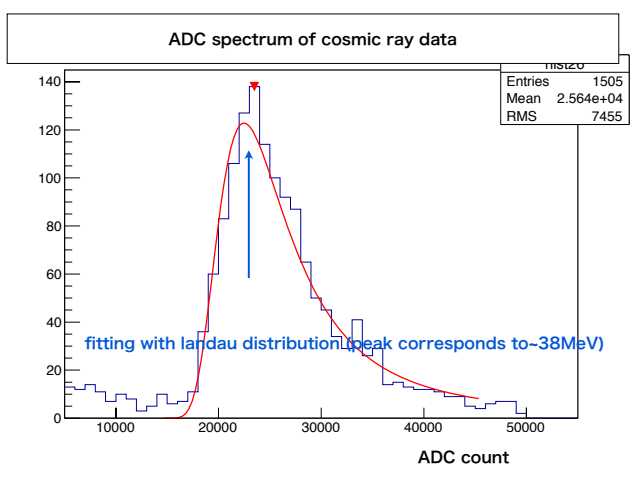

Figure 11: An example of ADC histogram of a cosmic- $\mu$ track

\section{References}

[1] Andrzej J. Buras, Selma Uhlig, and Felix Schwab. Waiting for precise measurements of $K^{+} \rightarrow \pi^{+} v \bar{v}$ and $K_{L}^{0} \rightarrow \pi^{0} v \bar{v}$. Rev. Mod. Phys., Vol. 80, No. 3, p. 965, 2008.

[2] J.K. Ahn et al. (E391a Collaboration), Phys. ReV. D 81 (2010) 072004. 